Herman Veenhof, Remco A. Koster, Lenneke A.T. Junier, Stefan P. Berger, Stephan J.L. Bakker and Daan J. Touw*

\title{
Volumetric absorptive microsampling and dried blood spot microsampling vs. conventional venous sampling for tacrolimus trough concentration monitoring
}

https://doi.org/10.1515/cclm-2019-1260

Received December 6, 2019; accepted April 27, 2020

\begin{abstract}
Objectives: Monitoring tacrolimus blood concentrations is important for preventing allograft rejection in transplant patients. Our hospital offers dried blood spot (DBS) sampling, giving patients the opportunity to sample a drop of blood from a fingerprick at home, which can be sent to the laboratory by mail. In this study, both a volumetric absorptive microsampling (VAMS) device and DBS sampling were compared to venous whole blood (WB) sampling.
\end{abstract}

Methods: A total of 130 matched fingerprick VAMS, fingerprick DBS and venous WB samples were obtained from 107 different kidney transplant patients by trained phlebotomists for method comparison using Passing-Bablok regression. Bias was assessed using Bland-Altman. A multidisciplinary team pre-defined an acceptance limit requiring $>80 \%$ of all matched samples within $15 \%$ of the mean of both samples. Sampling quality was evaluated for both VAMS and DBS samples.

Results: $32.3 \%$ of the VAMS samples and $6.2 \%$ of the DBS samples were of insufficient quality, leading to 88 matched samples fit for analysis. Passing-Bablok

\footnotetext{
* Corresponding author: Daan J. Touw, University of Groningen, Department of Clinical Pharmacy and Pharmacology, University Medical Center Groningen, PO Box 30.001, 9700 RB Groningen, The Netherlands; and University of Groningen, Department of Pharmaceutical Analysis, Groningen Research Institute of Pharmacy, University of Groningen, Groningen, The Netherlands,

Phone: +31 503614071, Fax: +31 503612417, E-mail: d.j.touw@umcg.nl Herman Veenhof and Lenneke A.T. Junier: University of Groningen, Department of Clinical Pharmacy and Pharmacology, University Medical Center Groningen, Groningen, The Netherlands

Remco A. Koster: University of Groningen, Department of Clinical Pharmacy and Pharmacology, University Medical Center Groningen, Groningen, The Netherlands; and PRA Health Sciences, Bioanalytical Laboratory, Assen, The Netherlands

Stefan P. Berger and Stephan J.L. Bakker: University of Groningen, Department of Internal Medicine, Division of Nephrology, University Medical Center Groningen, Groningen, The Netherlands
}

regression showed a significant difference between VAMS and WB, with a slope of 0.88 (95\% CI 0.81-0.97) but not for DBS (slope 1.00; 95\% CI 0.95-1.04). Both VAMS (after correction for the slope) and DBS showed no significant bias in Bland-Altman analysis. For VAMS and DBS, the acceptance limit was met for $83.0 \%$ and $96.6 \%$ of the samples, respectively.

Conclusions: VAMS sampling can replace WB sampling for tacrolimus trough concentration monitoring, but VAMS sampling is currently inferior to DBS sampling, both regarding sample quality and agreement with WB tacrolimus concentrations.

Keywords: dried blood spots; immunosuppressants; microsampling; therapeutic drug monitoring; volumetric absorptive microsampling.

\section{Introduction}

Therapeutic drug monitoring (TDM) of immunosuppressant drugs has been part of routine transplant patient care for decades. Sub-therapeutic dosing of immunosuppressants, such as tacrolimus, can lead to rejection of the allograft, while overdosing can lead to toxicity and sideeffects [1]. Because of great inter- and intra-individual variation in pharmacokinetics (PK), dosing of these drugs is tailored for each patient based on the blood drug concentration. This results in frequent patient visits to the hospital for venous blood sampling.

In the past years, several dried blood spot (DBS) microsampling methods for tacrolimus have been introduced, enabling patient home sampling [2-11]. Through a fingerprick, capillary blood is directly applied to special filter paper. After drying, the sample can be sent to the laboratory by mail. This decreases patient burden and allows more flexible immunosuppressant monitoring [8, 12]. Several of these DBS methods have shown to yield interchangeable results with venous whole blood (WB) and are routinely applied in transplant patient care, including in our hospital $[2,3,11,13]$. A drawback of DBS application is that sampling by the patient does not always lead

O Open Access. ( 2020 Daan J. Touw et al., published by De Gruyter. (cc)BY Public License. 
to sufficient quality DBS samples, and rates of up to $20 \%$ invalid samples for patient home sampled DBS have been reported [11, 14-16].

Volumetric absorptive microsampling (VAMS) was introduced as a potential successor of DBS sampling [17]. VAMS tips are designed to have several advantages compared to DBS. They wick-up an exact amount of sample volume (e.g. $20 \mu \mathrm{L}$ ) into a porous substrate, independent of hematocrit, and potentially improve the ease of sampling for the patient [17-19]. Although the effects of the hematocrit on the sample volume can be overcome by VAMS, this does not necessarily apply for the effect of hematocrit on extraction recovery from VAMS tips [20-23].

A recent study shows that tacrolimus can be reliably measured in VAMS throughout the complete dose interval of tacrolimus in renal transplant patients when comparing fingerprick VAMS (Mitra ${ }^{\circledR}$ ) results to paired venous WB samples [24]. However, in the latter study, the sample quality of VAMS was not discussed. In addition, there are no studies that directly compare the performance of fingerprick VAMS to fingerprick DBS for immunosuppressants. Only one study exists where fingerprick VAMS (Mitra ${ }^{\circledR}$ ) samples and fingerprick glass capillary tube samples (Drummond Aqua-Cap ${ }^{\circledR}$ ) were compared to venous WB samples for the drug radiprodil showing an underestimation of radiprodil exposure in VAMS (but not for capillary tube sampling) compared to venous WB [25].

In the current study, we compared both a novel VAMS sampling device (Mitra ${ }^{\circledR}$ ) and conventional DBS sampling to venous WB sampling with regard to interchangeability of analytical results and sample quality.

\section{Materials and methods}

\section{Training of phlebotomists}

For the DBS sampling, all phlebotomists were trained at the time DBS sampling was introduced (2016). At that time, the training consisted of a 15-min lecture explaining the sampling procedure, including common pitfalls and how to avoid them.

Because VAMS sampling was new in our hospital, the same phlebotomists were trained specifically for the VAMS sampling procedure. Although individual training of phlebotomists, including performing the sampling method themselves, is preferred, this was not feasible for one study coordinator for approximately 75 phlebotomists [26, 27]. Therefore, similar to the previous DBS validation studies performed in our hospital, all phlebotomists were trained in a 15-min lecture explaining the sampling procedure, including common pitfalls and how to avoid them based on information provided by literature and the manufacturer of the VAMS tips (Neoteryx, Torrance, CA, USA) $[2,13,19,28]$. An analysis was performed to evaluate if a learning effect over time could be observed on VAMS sampling. The percentage of sufficient quality tips for the first half of the samples was compared to the percentage of sufficient quality tips for the last half of the samples.

\section{Ethical approval, patients, sample collection and sample quality}

Patient samples were collected from tacrolimus-using adult kidney transplant patients during routine visits to the University Medical Center Groningen (UMCG, the Netherlands) for nephrologist consultation and TDM. Because of the nature of this study, the need to provide written informed consent by the patients was waived by the Ethics Committee of the UMCG (Metc 2011.394). This research was conducted in accordance with the Declaration of Helsinki and the EMA guidelines for good clinical practice E6(R2) [29]. All matched samples were obtained within $10 \mathrm{~min}$ of each other by the same phlebotomist following written instructions available at the time of sampling. First, the WB sample was obtained. Afterwards, a fingerpick was performed, and a DBS sample was obtained by letting two drops of blood fall freely on a Whatmann DMPK-C cards (GE Healthcare, Chicago, IL, USA) following a previously described method [27]. From the same fingerpick, two $20-\mu \mathrm{L}$ VAMS tips (Mitra ${ }^{\circledR}$, Neoteryx) were filled according to the manufacturer's instructions. Because the WB samples were part of routine care, they were analyzed within a day. After receiving the DBS and VAMS samples, they were inspected independently by two experienced lab technicians for quality, based on predefined criteria described earlier [15, 27, 30, 31]. If the judgment of the technicians differed, consensus was obtained by discussing each other's judgment. The DBS and VAMS samples were dried for at least $24 \mathrm{~h}$ at room temperature and packed in sealed plastic bags with a desiccant. The samples were stored at $-20{ }^{\circ} \mathrm{C}$ until analysis was performed. Stability of tacrolimus in DBS samples was validated for 29 weeks and in VAMS samples for 50 days at $-20^{\circ} \mathrm{C}$, so analysis occurred within these timeframes, respectively [23, 32, 33].

\section{Equipment and procedures}

Hematocrit of the WB samples was measured using a XN10/ XN20 hematology analyzer (Sysmex, Kobe, Japan).

Tacrolimus concentrations were analyzed in EDTA anti-coagulated WB samples using a validated analysis method on a Thermo Fisher Scientific triple quadrupole Quantiva MS/MS system with a Thermo Fisher Scientific Vanquish UPLC system (Waltham, MA, USA) [34]. Tacrolimus DBS samples were analyzed using a validated method on the aforementioned Thermo Fisher Scientific LC-MS/MS system [32, 33, 35]. The VAMS samples were analyzed for tacrolimus using a validated method on the aforementioned Thermo Fisher Scientific LC-MS/MS system [23]. The main difference between VAMS and DBS extraction, besides the need to manually punch the DBS samples, is the two-step extraction for VAMS samples where first 60:40 methanol:water is added to redissolve the red blood cells. Afterwards, methanol is added (step 2) to extract the analytes. For DBS, only one extraction solvent (80:20 methanol:water) is used [23, 33]. For liquid WB samples, tacrolimus is extracted using only methanol. Zinc sulfate is added during extraction for additional protein 
precipitation. Additional information regarding the VAMS, DBS and WB analysis methods, such as information on calibrators, standards, imprecision, extraction procedure, internal standard addition and traceability can be found in Supplement 1 .

\section{Statistical analysis}

Clinical validation was performed based on relevant guidelines by the CLSI, FDA, EMA and the recently published Guideline on Development and Validation of Dried Blood Spot-Based Methods for Therapeutic Drug Monitoring [27, 36-38]. In short, method comparison was performed using the Passing-Bablok regression analysis [39]. The Bland-Altman analysis was used to calculate bias [40]. The limit of clinical acceptance was set $a$ priori at $85 \%-115 \%$ around the ratio of matched WB-DBS and matched WB-VAMS samples for at least $80 \%$ of the samples in accordance with earlier studies [13, 27]. These limits were chosen in a multidisciplinary team consisting of transplantation nephrologists, pharmacists and analysts and were based on current trough concentration targets and the relevant concentration window for tacrolimus in kidney transplantation in combination with the aspects of the analytical method used for VAMS, DBS and WB $[1,13,32-35]$. The predictive performance of both the DBS and VAMS method was established using the method described by Sheiner and Beal [41]. In short, WB concentrations were predicted from both DBS and VAMS concentrations according to a previously described method $[3,13,27]$. The bias of the prediction is the median difference between the predicted and true concentration and is shown by the median prediction error (MPE) and the median percentage prediction error (MPPE). The imprecision is the variance of the predicted values which is measured by the root median squared prediction error (RMSE) and the median absolute percentage prediction error (MAPE). The following equations were used:

Median Prediction Error $(M P E)=$ median $($ Predicted WB - WB $)$

Median Percentage Prediction Error(MPPE)

$$
=\operatorname{median}\left(100 \% * \frac{\text { Predicted WB }-\mathrm{WB}}{\mathrm{WB}}\right)
$$

Root Median Squared Prediction Error (RMSE) $=$ $\sqrt{\text { Median }}(\text { Predicted WB }- \text { WB })^{2}$

$$
\begin{aligned}
& \text { Median Absolute Percentage Prediction Error(MAPE) } \\
& =\operatorname{median}\left(100 \% * \frac{\mid \text { Predicted } \mathrm{WB}-\mathrm{WB} \mid}{\mathrm{WB}}\right)
\end{aligned}
$$

In accordance with other studies, acceptable values for MPPE and MAPE were set at $<15 \%$ and at least $67 \%$ of all samples should have an absolute prediction error of $<20 \%[3,6,13,42]$. Statistical analysis was performed using Analyse-it ${ }^{\circledR}$ Method Validation Edition for Microsoft Excel version 4.18.6 (Analyse-it, Leeds, UK) and Microsoft Excel 2010 (Microsoft Inc., Redmond, WA, USA). Normality was tested using a Shapiro-Wilk test. All categorical data were expressed as percentages. Numeric data were expressed as mean \pm standard deviation (SD) and range when normally distributed, or as median with interquartile range (IQR) and range when not normally distributed.

\section{Results}

\section{Sample quality}

In total, 130 matched samples were obtained from 107 adult kidney transplant patients between June 2018 and October 2018. For the VAMS samples, 42 (32.3\%) of the samples were rejected because of insufficient quality, 26 samples $(20.0 \%)$ contained one sufficient quality tip and 62 samples (47.7\%) contained two sufficient quality tips. Consensus between technicians was needed for eight (6.2\%) of the VAMS samples. Three reasons for VAMS sample rejection were identified: (1) for 31 individual tips, the tip touched the cap of the sampling container caused by improper closing of the cap (Figure 1B); (2) for 30 individual tips, the tip was oversaturated, caused by letting blood fall on the tip instead of dipping the tip in the blood (Figure 1C); (3) for 39 individual tips, the tip was undersaturated, caused by a too small amount of blood obtained from the fingerprick or not dipping the tip into the blood long enough (Figure 1D). When comparing the VAMS sample quality for the first half of the samples to the last half of the samples, no learning effect was observed (63.8\% and $66.9 \%$ samples of sufficient quality, respectively). For the DBS samples, eight samples (6.2\%) were rejected because of insufficient quality, 23 samples (17.7\%) contained one sufficient quality spot and 99 (76.2\%) of the samples contained two sufficient quality spots.

\section{Patients}

In total, 88 matched samples from 72 unique patients were included in the method comparison analysis. Patient demographics are summarized in Table 1. The median concentrations of tacrolimus in WB, DBS and VAMS can be found in Table 2. Average hematocrit was 0.39 with a SD of 0.05 and a range of 0.25-0.50. All tacrolimus concentrations were within the analytically validated range. All hematocrit values were within the analytically validated range.

\section{Clinical validation of VAMS}

The Passing-Bablok fit was $\mathrm{y}=0.88 \mathrm{x}+0.01$ (95\% CI slope, 0.81-0.97; 95\% CI intercept, -0.47-0.39) showing no significant constant difference. A significant systematic difference of $12 \%$ lower tacrolimus concentration in VAMS compared to WB was observed (Figure 2). This systematic 


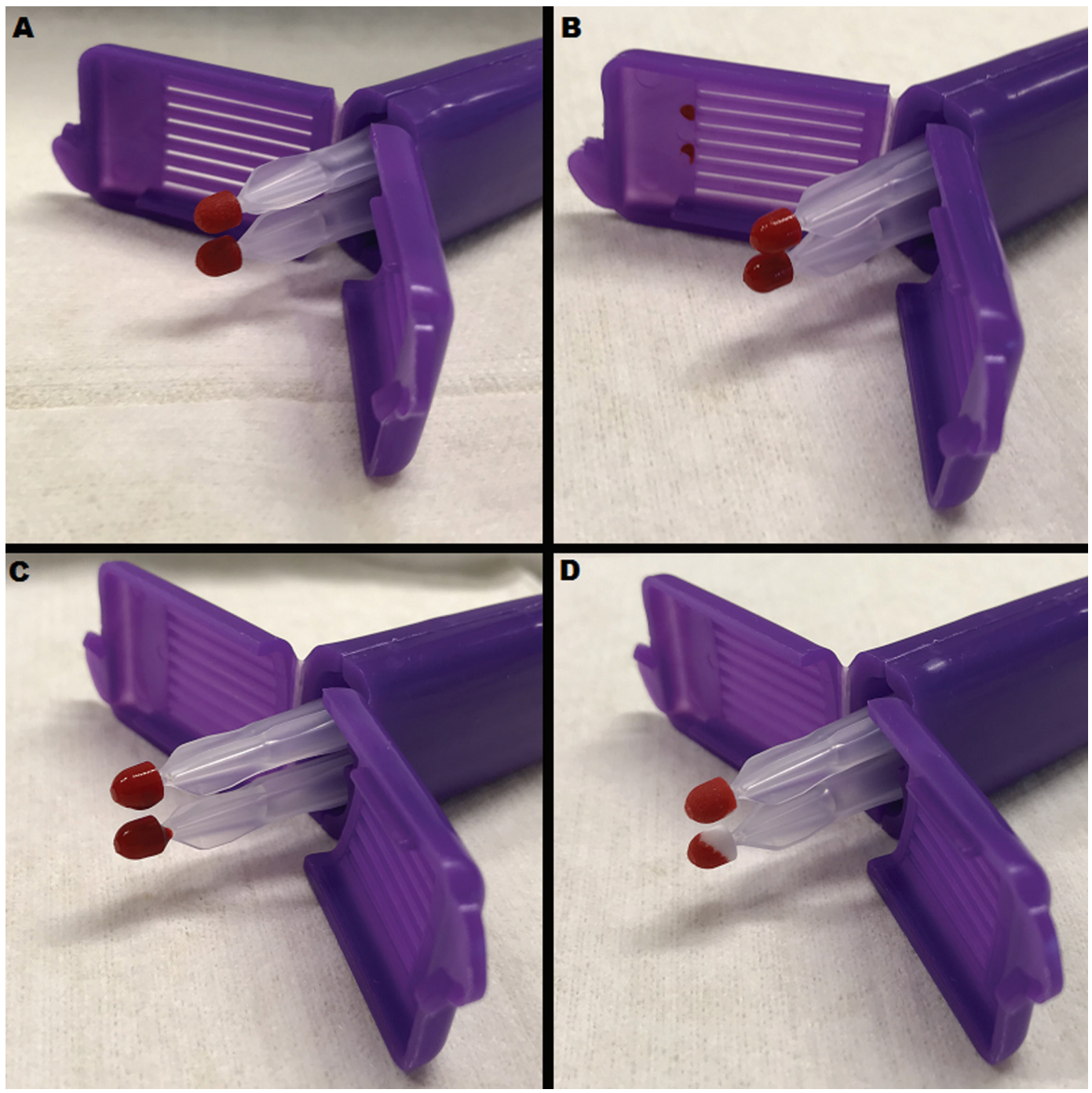

Figure 1: Different types of quality in $20 \mu \mathrm{L}$ volumetric absorptive microsampling (VAMS) samples.

(A) Sufficient quality VAMS sample meeting all requirements. (B) Insufficient quality VAMS sample because the containers' cap touched the tip, blood is visible on the inside of the cap. (C) Insufficient quality VAMS sample because of oversaturation, blood is visible on the tip holder. (D) Insufficient quality VAMS sample due to undersaturation, the tip is not completely filled with blood.

Table 1: Patient demographics.

\begin{tabular}{lll}
\hline $\begin{array}{l}\text { Patient } \\
\text { demographics }\end{array}$ & $\mathbf{n}$ & Median (range) \\
\hline Age, years & 72 & $58(21-78)$ \\
Sex & 72 & $\begin{array}{l}42 \text { male }(58.3 \%) \\
30 \text { female (41.7\%) } \\
\text { Time since }\end{array}$ \\
transplantation & 72 & $\begin{array}{l}\text { 1 year, 7 months, 25 days } \\
\text { (22 days-16 years, 4 months) }\end{array}$ \\
\hline
\end{tabular}

difference was used to derive the following conversion formula: [tacrolimus WB concentration] $=$ [tacrolimus VAMS concentration]/0.88. This conversion formula was used to recalculate all VAMS values, and these
Table 2: Median tacrolimus concentrations including IQR and range.

\begin{tabular}{lcr}
\hline Concentration & $\mathbf{n}$ & Median [IQR] (range) \\
\hline Tacrolimus in WB, $\mu \mathrm{g} / \mathrm{L}$ & 88 & $6.2[4.8-8.3](3.0-24.3)$ \\
Tacrolimus in DBS, $\mu \mathrm{g} / \mathrm{L}$ & 88 & $6.2[4.8-8.3](2.8-23.5)$ \\
Tacrolimus in VAMS, $\mu \mathrm{g} / \mathrm{L}$ & 88 & $6.2[4.8-8.2](2.8-17.9)$ \\
\hline
\end{tabular}

DBS, dried blood spot; IQR, interquartile range; VAMS, volumetric absorptive microsampling; WB, whole blood.

recalculated values were used in Bland-Altman analysis [27]. No significant bias was found in Bland-Altman analysis, with a mean WB/VAMS ratio of 1.00 (95\% CI 0.981.02) as shown in Figure 2. In total, $83.0 \%$ of the matched 

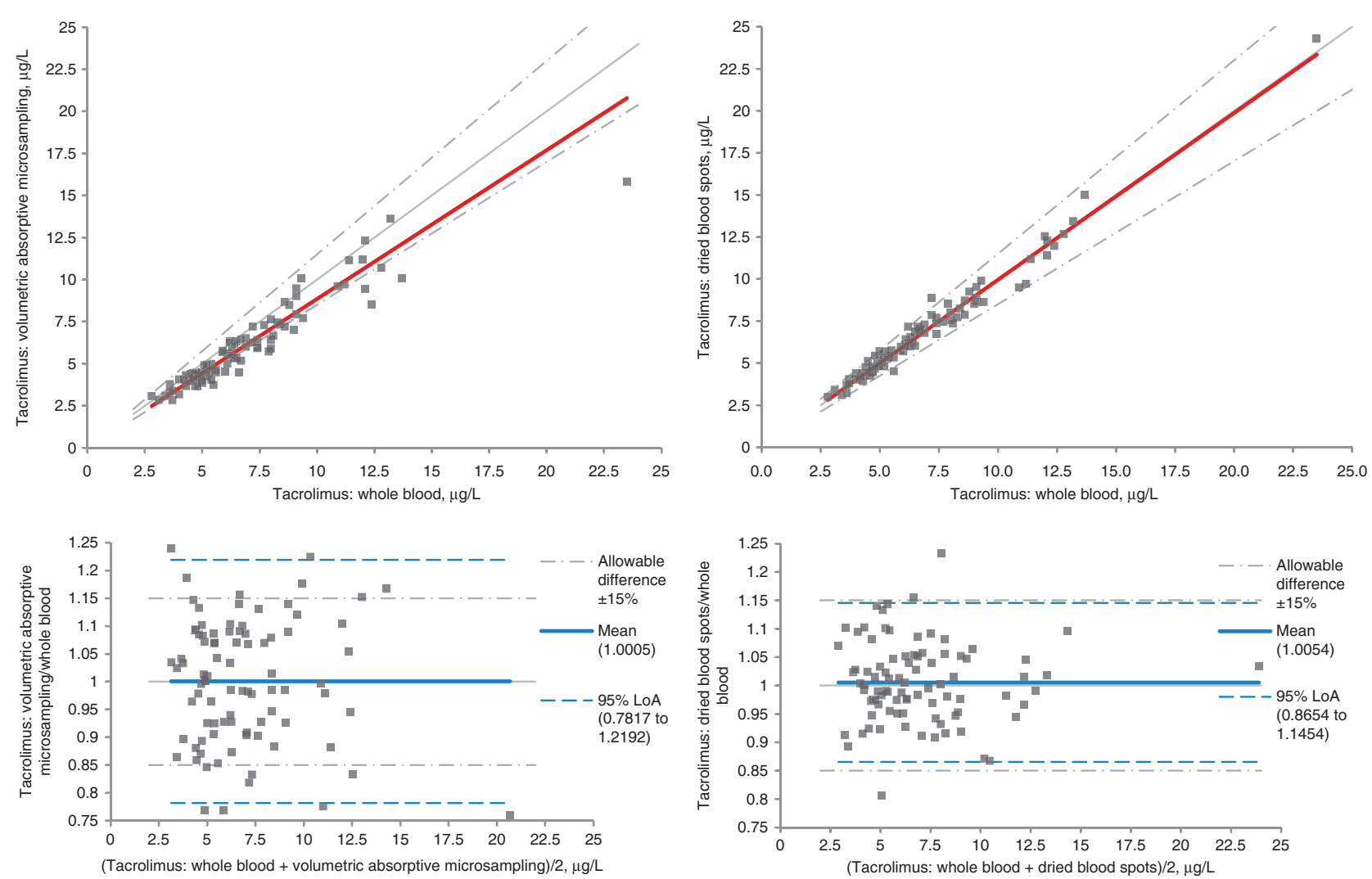

Figure 2: Method comparison between whole blood (WB) tacrolimus levels, volumetric absorptive microsampling (VAMS) tacrolimus levels and dried blood spot (DBS) tacrolimus levels for 88 matched samples.

In the upper left panel, the bold red continuous line is the Passing-Bablok regression line $\mathrm{y}=0.88 \mathrm{x}+0.01(95 \% \mathrm{Cl}$ slope, $0.81-0.97 ; 95 \%$ $\mathrm{Cl}$ intercept, $-0.47-0.39)$ for WB vs. VAMS. The dotted/dashed line is the $15 \%$ limit of clinical acceptance. In the upper right panel, the bold red continuous line is the Passing-Bablok regression line $\mathrm{y}=0.99 \mathrm{x}+0.02$ (95\% Cl slope, $0.95-1.04 ; 95 \% \mathrm{Cl}$ intercept, $-0.26-0.28)$ for WB vs. DBS. The dotted/dashed line is the $15 \%$ limit of clinical acceptance. The lower left panel shows the Bland-Altman analysis bias estimation based on recalculated values for VAMS using the formula [tacrolimus WB concentration] = [tacrolimus VAMS concentration]/0.88. Calculated bias is 1.00 ( $95 \% \mathrm{Cl} 0.98-1.02)$. The dotted/dashed line is the $15 \%$ limit of clinical acceptance. The dashed line is the $95 \%$ limits of agreement (LoA). The lower right panel shows the Bland-Altman analysis bias estimation for WB vs. DBS of 1.01 (95\% Cl 0.99-1.02). The dotted/dashed line is the $15 \%$ limit of clinical acceptance. The dashed line is the $95 \%$ LoA.

samples are within the limits of clinical acceptance meeting the requirement of at least $80 \%$. Because of the correction factor used, the bias estimation in the predictive performance was small with an MPE of $0.00 \mu \mathrm{g} / \mathrm{L}$ and an MPPE of $0.00 \%$. The predictive performance of imprecision as shown by the RMSE was small with a value of $0.54 \mu \mathrm{g} / \mathrm{L}$. The MAPE was within acceptable limits $(<15 \%)$ with a value of $8.74 \%$. The acceptance limit for MAPE ( $>67 \%$ of samples with a value $<20 \%$ ) was met with 82 out of 88 samples (93.2\%) (Figure 3).

\section{Clinical validation of DBS}

The Passing-Bablok fit was $\mathrm{y}=0.99 \mathrm{x}+0.02(95 \% \mathrm{CI}$ slope, 0.95-1.04; 95\% CI intercept, $-0.26-0.28$ ) showing no significant systematic or constant difference between WB and DBS as shown in Figure 2. Bland-Altman analysis showed no significant bias, with a mean WB/DBS ratio of 1.01 (95\% CI 0.99-1.02) as shown in Figure 2. The 95\% limits of agreement (LoA) are within the limits of clinical acceptance set at $\pm 15 \%$. In total, $96.6 \%$ of the matched samples are within the limits of clinical acceptance meeting the requirement of at least $80 \%$. The bias estimation in the predictive performance was small with an MPE of $0.00 \mu \mathrm{g} / \mathrm{L}$ and an MPPE of $-0.04 \%$. The predictive performance of imprecision as shown by the RMSE was small with a value of $0.32 \mu \mathrm{g} / \mathrm{L}$. The MAPE was within acceptable limits $(<15 \%)$ with a value of $5.18 \%$. The acceptance limit for MAPE ( $>67 \%$ of samples with a value $<20 \%$ ) was met with 87 out of 88 samples (98.9\%) (Figure 3). 


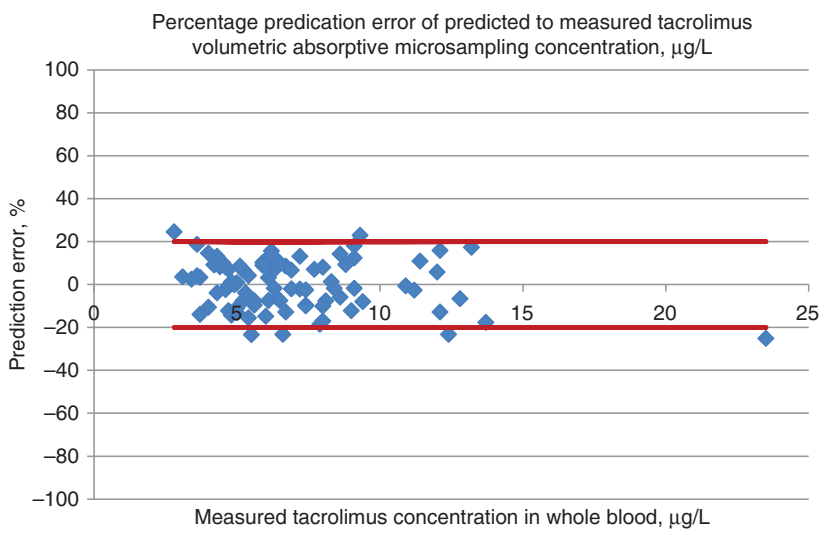

Percentage predication error of predicted to measured tacrolimus DBS concentration, $\mu \mathrm{g} / \mathrm{L}$

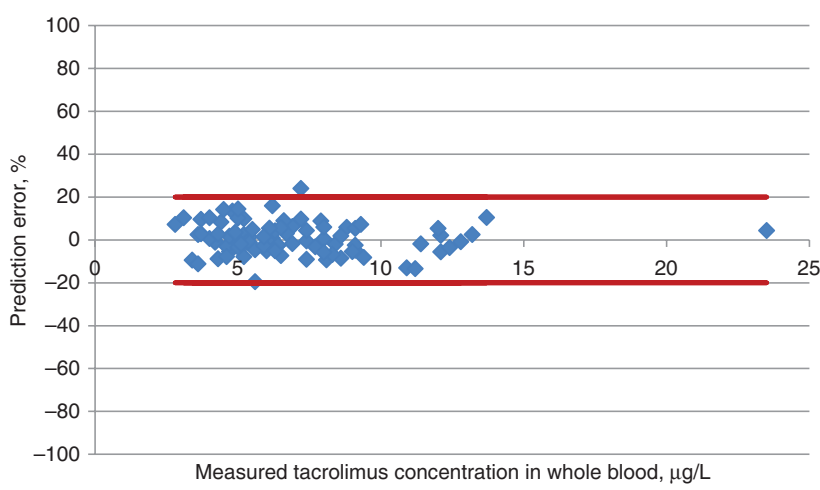

Figure 3: Predictive performance of calculating whole blood (WB) tacrolimus concentrations from both volumetric absorptive microsampling (VAMS) samples and dried blood spot (DBS) samples. The upper panel shows the percentage prediction error of predicted to measured tacrolimus volumetric absorptive microsampling (VAMS) concentrations with acceptable prediction error set at $-20 \%$ and $20 \%$ after applying the formula [tacrolimus WB concentration $]=[$ tacrolimus VAMS concentration $] / 0.88$. The lower panel shows the percentage prediction error of predicted to measured tacrolimus dried blood spots (DBS) concentrations with acceptable prediction error set at $-20 \%$ and $20 \%$.

\section{Discussion}

This study showed good agreement between tacrolimus VAMS and tacrolimus WB concentrations, and very good agreement between tacrolimus DBS and tacrolimus WB concentrations in kidney transplant patients over a relevant range of trough concentrations. The predictive performance of both the VAMS and DBS meet the predefined criterion. Both VAMS (after correction) and DBS meet the predefined limits of clinical acceptance and can be used in transplant patient care.

The conclusion that DBS performs better than VAMS was unexpected. We considered that this might be caused by the fact that DBS sampling has been in use for over 3 years in our hospital, allowing quality of DBS sampling and DBS analysis to improve. In our previous validation studies, performed prior to DBS implementation in routine care, no limits of clinical acceptance were set [2,33]. In order to get more insight into the performance during the early adoptive phase of DBS, we applied the limits of clinical acceptance used in this study to the data of the previous studies and show that these limits would not be met (respectively 78.9\% [ $n=82 / 104]$ [2] and 80.0\% [ $n=70 / 85]$ ) [33]. The fact that the performance of the DBS assay has improved over time could be attributed to improvements in DBS sampling and/or DBS analysis methods or even the WB analysis which is used as the gold standard.

During VAMS analytical validation, recovery of tacrolimus was stable across a wide hematocrit range $(0.20-0.60 \mathrm{v} / \mathrm{v})$ and concentration range $(3.0 \mu \mathrm{g} / \mathrm{L}-40$ $\mu \mathrm{g} / \mathrm{L})$, with a maximum bias of $-8.3 \%$ at extreme values for hematocrit and tacrolimus concentrations (respectively $0.20 \mathrm{v} / \mathrm{v}$ and $40 \mu \mathrm{g} / \mathrm{L}$ ) [23]. Therefore, it was unexpected that the VAMS method showed a significant systematic difference of $12 \%$ lower tacrolimus concentration in VAMS compared to WB samples.

Because of insufficient sample quality, only 62 duplicate VAMS samples were available for analysis [27]. Method comparison using the mean value of the duplicate samples yielded a similar conversion formula for VAMS in PassingBablok analysis and similar bias in Bland-Altman analysis (data not shown). It can thus be concluded that duplicate VAMS sample analysis has no positive effect on the quality of the analysis results and has no added benefit.

Other studies report both lower and higher concentrations in VAMS compared to WB for various drugs [20, 25]. The study by Kita et al. reported an average of $14 \%$ higher AUC for tacrolimus in rat tail blood collected in VAMS compared to wet rat tail blood samples [43]. In the study by Vethe et al., who performed a clinical validation study for tacrolimus with paired WB and VAMS samples from two full 12-h PK curves of 27 adult renal transplant patients totaling 679 matched samples of which 105 were trough concentrations, no significant systematic differences are observed between WB and VAMS samples for tacrolimus across the entire concentration and hematocrit range [24]. We consider three possible explanations for the lower concentrations of tacrolimus in VAMS compared to WB in our study. The first is the possible influence of the anticoagulant on the analytical results [27]. During method validation and sample analysis for this study, citrate anti-coagulated blood was used for the calibration and quality control (QC) samples for both the DBS and VAMS samples [23, 33]. The obtained patient VAMS and DBS samples consisted of capillary blood which does not contain an anti-coagulant, and the WB 
samples were anti-coagulated with EDTA. Although this proves to have no influence on DBS analytical results, the absence of the citrate anticoagulant in patient samples might lower the VAMS extraction recovery. It is interesting to see Vethe et al. describe the use of water as the first extraction solvent while other studies used organic extraction solvents (e.g. methanol or methanol/ water) $[20,21,23,24,43]$. The application of pure water as the first added extraction solvent might overcome the potential effects of anti-coagulants from the VAMS tips. However, Vethe et al. did not specify the anticoagulant of the blood used during method validation and patient sample analysis [24]. The second reason might be the batch-to-batch differences in blood wicking volume of the Mitra ${ }^{\circledR}$ tips. However, we observed only a difference of $3 \%$ lower blood wicking volume in the batch of VAMS tips used for patient sampling compared to the batch of VAMS tips used during method development and validation, according to the certificates of conformance. The third reason might be the influence of 'invisible undersampling' of VAMS samples. Oversaturated VAMS tips will all be identified and excluded from analysis. Although obviously undersaturated VAMS tips (see Figure 1D) will be identified and excluded, this might not be the case for slightly undersaturared VAMS tips. According to the sampling instruction, the VAMS tip should remain in the drop of fingerprick blood for $2 \mathrm{~s}$ after the tip turns completely red to allow the complete filling of the inside of the tip [28]. When removed earlier, the tip might not be completely filled with blood, without the possibility of identifying this during sample inspection. To investigate this, we assumed that, for samples that passed QC where the values of the two duplicate VAMS tips differed $>10 \%$ compared to the mean of both samples, this was caused by invisible undersaturation. We assumed that only the higher of these two values would represent a properly saturated tip. This was the case for 17/62 samples. When using only the highest values in the Passing-Bablok analysis, we still found a 7\% lower concentration of tacrolimus in VAMS compared to WB. Combined with the 3\% lower blood wicking volume a difference of $4 \%$ lower tacrolimus concentration in VAMS compared to WB remains, which might be attributed to the earlier mentioned effect of the anticoagulant combined with the extraction method.

When using the aforementioned conversion formula to calculate VAMS tacrolimus concentrations, the results from this study are comparable to the results of the study by Vethe et al. In their study, a limit of clinical acceptance of $20 \%$ was defined [24]. In total, $97.1 \%$ of the trough concentration samples $(n=105)$ was within this limit. If a limit for clinical acceptance of $20 \%$ was applied to our study, $94.3 \%$ of the VAMS samples would be within this limit.

The rejection rate of $32.3 \%$ for the VAMS samples was unexpected. Phlebotomists were trained using a similar training method that was used for the previous DBS clinical validation studies performed in our hospital. In these previous studies, rejection rates of DBS samples were $0.0 \%-4.8 \%[2,13,33]$. Possible explanations for the high VAMS rejection rate can be as follows: (1) letting drops of blood fall on the VAMS tip instead of absorbing the blood, because phlebotomists might be used to the free-falling drop of blood in DBS sampling (Figure 1C); (2) not enough blood from a single fingerprick to obtain a VAMS sample after a DBS sample might explain undersaturation (Figure 1D); (3) touching the blood sample by improper closing of the lids of the purple Mitra ${ }^{\circledR}$ cartridge (Figure 1B). In the study by Vethe et al., no data were provided on sample quality of VAMS tips [24]. Although their study did not state how many phlebotomists obtained the samples or how they were trained, it is likely that only a limited number of study coordinators obtained the samples because it was a full-curve PK study. Involving only a few study coordinators whose training included practicing all steps of the sampling method can lead to up to $100 \%$ sufficient quality samples [26]. In our hospital, a total of 75 different phlebotomists could have performed the VAMS sampling. It can be concluded that training is of essence in order to ensure acceptable sample quality. Even experience of phlebotomists with other microsampling techniques such as DBS seems to be of no guarantee for good quality VAMS samples.

Although meeting the predefined limits of clinical acceptance, at this moment VAMS results are inferior to DBS results, regarding agreement with WB results. In addition, introduction of VAMS sampling would likely not improve the amount of sufficient quality samples produced by patients at home. As a consequence, conventional DBS home sampling by transplant patients is currently the preferred microsampling method in our hospital for TDM of tacrolimus.

In future clinical validation studies, sample acquisition by only a limited number of well-trained personnel is key in obtaining high-quality samples. The training method itself might be subject to assessment and include a practical test before staff members are allowed to obtain samples. In addition, studies are needed where patients perform both DBS and VAMS sampling in order to assess the true difference in sample quality and patients' sampling method preference. In such a study, the costs for both VAMS and DBS home sampling should also be assessed. 
Acknowledgments: We would like to thank the phlebotomists of the 'prikpoli' of the UMCG for obtaining the dried blood spot and volumetric absorptive microsampling samples.

Research funding: This work was supported by the Netherlands Organization for Health Research and Development (ZonMw, The Hague, Netherlands) grant 836044004, Funder Id: http://dx.doi.org/10.13039/501100001826. This study was performed using the infrastructure and data provided by the TransplantLines Biobank and Cohort Study, which is registered at ClinicalTrials.gov under identifier NCT03272841.

Author contributions: All authors have accepted responsibility for the entire content of this manuscript and approved its submission.

Competing interests: Authors state no conflict of interest. Ethical approval: Because of the nature of this study, the need to provide written informed consent by the patients was waived by the Ethics Committee of the UMCG (Metc 2011.394). This research was conducted in accordance with the Declaration of Helsinki and the EMA guidelines for good clinical practice E6(R2).

\section{References}

1. Brunet $M$, van Gelder T, Åsberg A, Haufroid V, Hesselink DA, Langman L, et al. Therapeutic drug monitoring of tacrolimuspersonalized therapy: second consensus report. Ther Drug Monit 2019;41:261-307.

2. Veenhof H, Koster RA, Alffenaar JW, Berger SP, Bakker SJ, Touw DJ. Clinical validation of simultaneous analysis of tacrolimus, cyclosporine $A$ and creatinine in dried blood spots in kidney transplant patients. Transplantation 2017;101:1727-33.

3. Zwart TC, Gokoel SR, van der Boog PJ, de Fijter JW, Kweekel DM, Swen JJ, et al. Therapeutic drug monitoring of tacrolimus and mycophenolic acid in outpatient renal transplant recipients using a volumetric dried blood spot sampling device. Br J Clin Pharmacol 2018;84:2889-902.

4. Webb NJA, Roberts D, Preziosi R, Keevil BG. Fingerprick blood samples can be used to accurately measure tacrolimus levels by tandem mass spectrometry. Pediatr Transplant 2005;9:729-33.

5. van Boekel GA, Donders AR, Hoogtanders KE, Havenith TR, Hilbrands LB, Aarnoutse RE. Limited sampling strategy for prolonged-release tacrolimus in renal transplant patients by use of the dried blood spot technique. Eur J Clin Pharmacol 2015;71:811-6.

6. Martial LC, Hoogtanders KE, Schreuder MF, Cornelissen EA, van der Heijden J, Joore MA, et al. Dried blood spot sampling for tacrolimus and mycophenolic acid in children: analytical and clinical validation. Ther Drug Monit 2017;39:412-21.

7. Leino AD, King EC, Jiang W, Vinks AA, Klawitter J, Christians U, et al. Assessment of tacrolimus intra-patient variability in stable adherent transplant recipients: establishing baseline values. Am J Transplant 2018;19:1410-20.
8. Hoogtanders K, van der Heijden J, Christiaans M, Edelbroek P, van Hooff JP, Stolk LM. Therapeutic drug monitoring of tacrolimus with the dried blood spot method. J Pharm Biomed Anal 2007;44:658-64.

9. Hinchliffe E, Adaway J, Fildes J, Rowan A, Keevil BG. Therapeutic drug monitoring of ciclosporin $A$ and tacrolimus in heart lung transplant patients using dried blood spots. Ann Clin Biochem 2014;51(Pt 1):106-9.

10. Dickerson JA, Sinkey M, Jacot K, Stack J, Sadilkova K, Law YM, et al. Tacrolimus and sirolimus in capillary dried blood spots allows for remote monitoring. Pediatr Transplant 2015;19:101-6.

11. Al-Uzri AA, Freeman KA, Wade J, Clark K, Bleyle LA, Munar M, et al. Longitudinal study on the use of dried blood spots for home monitoring in children after kidney transplantation. Pediatr Transplant 2017;21. doi: 10.1111/petr.12983.

12. Martial LC, Aarnoutse RE, Schreuder MF, Henriet SS, Brüggemann RJ, Joore MA. Cost evaluation of dried blood spot home sampling as compared to conventional sampling for therapeutic drug monitoring in children. PLoS One 2016;11:e0167433.

13. Veenhof H, Koster RA, Alffenaar JC, van den Berg AP, de Groot $M R$, Verschuuren EA, et al. Clinical application of a dried blood spot assay for sirolimus and everolimus in transplant patients. Clin Chem Lab Med 2019;57:1854-62.

14. Boons CC, Timmers L, Janssen JJ, Swart EL, Hugtenburg JG, Hendrikse NH. Feasibility of and patients' perspective on nilotinib dried blood spot self-sampling. Eur J Clin Pharmacol 2019;75:825-9.

15. Zuur MA, Veenhof H, Aleksa A, van't Boveneind-Vrubleuskaya N, Darmawan E, Hasnain MG, et al. Quality assessment of dried blood spots from tuberculosis patients from four countries. Ther Drug Monit 2019;41:714-8.

16. Veenhof H, Koster RA, Brinkman R, Senturk E, Bakker SJ, Berger $\mathrm{SP}$, et al. Performance of a web-based application measuring spot quality in dried blood spot sampling. Clin Chem Lab Med 2019;57:1846-53.

17. Spooner N, Denniff P, Michielsen L, De Vries R, Ji QC, Arnold ME, et al. A device for dried blood microsampling in quantitative bioanalysis: overcoming the issues associated blood hematocrit. Bioanalysis 2015;7:653-9.

18. Kok MG, Fillet M. Volumetric absorptive microsampling: current advances and applications. J Pharm Biomed Anal 2018;147: 288-96.

19. Denniff P, Spooner N. Volumetric absorptive microsampling: a dried sample collection technique for quantitative bioanalysis. Anal Chem 2014;86:8489-95.

20. Verheijen R, Thijssen B, Atrafi F, Schellens J, Rosing H, de Vries $\mathrm{N}$, et al. Validation and clinical application of an LC-MS/MS method for the quantification of everolimus using volumetric absorptive microsampling. J Chromatogr B 2019;1104:234-9.

21. De Kesel PM, Lambert WE, Stove CP. Does volumetric absorptive microsampling eliminate the hematocrit bias for caffeine and paraxanthine in dried blood samples? A comparative study. Anal Chim Acta 2015;881:65-73.

22. Velghe $S$, Delahaye L, Stove CP. Is the hematocrit still an issue in quantitative dried blood spot analysis? J Pharm Biomed Anal 2019;163:188-96.

23. Koster RA, Niemeijer P, Veenhof H, Hateren Kv, Alffenaar JC, Touw DJ. A volumetric absorptive microsampling LC-MS/MS method for five immunosuppressants and their hematocrit effects. Bioanalysis 2019;11:495-508. 
24. Vethe NT, Gustavsen MT, Midtvedt K, Lauritsen ME, Andersen AM, Anders Åsberg M, et al. Tacrolimus can be reliably measured with volumetric absorptive capillary microsampling throughout the dose interval in renal transplant recipients. Ther Drug Monit 2019;41:607-14.

25. Sciberras D, Otoul C, Lurquin F, Smeraglia J, Lappert A, De Bruyn $S$, et al. A pharmacokinetic study of radiprodil oral suspension in healthy adults comparing conventional venous blood sampling with two microsampling techniques. Pharmacol Res Perspect 2019;7:e00459.

26. Panchal T, Spooner N, Barfield M. Ensuring the collection of high-quality dried blood spot samples across multisite clinical studies. Bioanalysis 2017;9:209-13.

27. Capiau S, Veenhof H, Koster R, Bergqvist Y, Boettcher M, Halmingh $\mathrm{O}$, et al. Official International Association for Therapeutic Drug Monitoring and Toxicology guideline: development and validation of dried blood spot-based methods for therapeutic drug monitoring. Ther Drug Monit 2019;41:409-30.

28. Protti M, Mandrioli R, Mercolini L. Tutorial: Volumetric absorptive microsampling (VAMS). Anal Chim Acta 2019;1046:32-47.

29. World Medical Association. World Medical Association Declaration of Helsinki: ethical principles for medical research involving human subjects. J Am Med Assoc 2013;310:2191-4.

30. CLSI. Blood collection on Filter Paper for Newborn Screening Programs; Approved Standard - Sixth Edition. CLSI Document NBS01-A6. Wayne, PA: Clinical and Laboratory Standards Institute, 2013;NBS01-A6.

31. World Health Organization. Participant Manual Module 14 Blood Collection and Handling - Dried Blood Spot (DBS). 2005; Module 14: EQA (December).

32. Koster RA, Alffenaar JW, Greijdanus B, Uges DR. Fast LC-MS/MS analysis of tacrolimus, sirolimus, everolimus and cyclosporin A in dried blood spots and the influence of the hematocrit and immunosuppressant concentration on recovery. Talanta 2013;115:47-54.

33. Koster RA, Veenhof H, Botma R, Hoekstra AT, Berger SP, Bakker SJ, et al. Dried blood spot validation of five immunosuppressants, without hematocrit correction, on two LC-MS/MS systems. Bioanalysis 2017;9:553-63.
34. Koster RA, Dijkers EC, Uges DR. Robust, high-throughput LC-MS/ MS method for therapeutic drug monitoring of cyclosporine, tacrolimus, everolimus, and sirolimus in whole blood. Ther Drug Monit 2009;31:116-25.

35. Koster RA, Greijdanus B, Alffenaar JC, Touw DJ. Dried blood spot analysis of creatinine with LC-MS/MS in addition to immunosuppressants analysis. Anal Bioanal Chem 2015;407:1585-94.

36. Food and Drug Administration, US Department of Health and Human Services. Guidance for Industry, Bioanalytical Method Validation. Rockville, MD, USA: Food and Drug Administration, US Department of Health and Human Services, 2018.

37. European Medicines Agency. Guideline on bioanalytical method validation. London, UK: European Medicines Agency, 2011.

38. CLSI. Measurement Procedure Comparison and Bias Estimation Using Patient Samples; Approved Guideline - Third Edition. CLSI document EP09-A3. Wayne, PA: Clinical and Laboratory Standards Institute, 2013.

39. Passing H, Bablok W. A New Biometrical Procedure for Testing the Equality of Measurements from Two Different Analytical Methods. Application of linear regression procedures for method comparison studies in Clinical Chemistry, Part I. Clin Chem Lab Med 1983;21:709-20.

40. Bland J, Altman D. Statistical methods for assessing agreement between two methods of clinical measurement. The Lancet 1986;327:307-10.

41. Sheiner LB, Beal SL. Some suggestions for measuring predictive performance. J Pharmacokinet Biopharm 1981;9:503-12.

42. Willemsen A, Knapen L, de Beer Y, Brüggemann R, Croes S, van Herpen C, et al. Clinical validation study of dried blood spot for determining everolimus concentration in patients with cancer. Eur J Clin Pharmacol 2018;74:465-71.

43. Kita K, Noritake K, Mano Y. Application of a volumetric absorptive microsampling device to a pharmacokinetic study of tacrolimus in rats: comparison with wet blood and plasma. Eur J Drug Metab Pharmacokinet 2019;44:91-102.

Supplementary Material: The online version of this article offers supplementary material (https://doi.org/10.1515/cclm-2019-1260). 